\title{
Calidad en la Educación e Infraestructuras del Transporte desde la Accesibilidad Territorial. Departamento de Caldas en Colombia como Caso de Estudio
}

\author{
Juan D. Zuluaga ${ }^{(1)}$, Diego A. Escobar ${ }^{(2)}$, Juan D. Hincapié(3) \\ (1) Facultad de Ingeniería y Arquitectura, Universidad Nacional de Colombia Sede Manizales. Colombia. \\ (e-mail: judzuluagaga@unal.edu.co) \\ (2) Facultad de Ingeniería y Arquitectura, Universidad Nacional de Colombia Sede Manizales. Colombia. \\ (e-mail: daescobarga@unal.edu.co) \\ (3) Facultad de Ingeniería, Universidad Tecnológica de Pereira, Colombia. \\ (e-mail: judaz@sirius.utp.edu.co)
}

Recibido Dic. 21, 2016; Aceptado Mar. 2, 2017; Versión final Abr. 20, 2017, Publicado Dic. 2017

\begin{abstract}
Resumen
En esta investigación se establece la relación existente entre las características operativas de la red de infraestructura del transporte del Departamento de Caldas (Colombia) y los resultados de las pruebas SABER-11 de las instituciones educativas obtenidas en el año 2015. Estas pruebas son las que las instituciones de educación superior toman como referencia para los procesos de admisión. Se calcula la cobertura geográfica para todos los colegios a partir de la red de infraestructuras del transporte y se analizan los resultados según la calificación obtenida en las pruebas por cada Institución Educativa. Los resultados obtenidos permitieron establecer que existe una relación directa entre la calidad de la educación ofrecida y las condiciones de accesibilidad territorial hacia las instituciones educativas del Departamento, en términos de los tiempos medios de viaje que deben ser invertidos. La investigación concluye sobre la importancia de tener instituciones educativas con altas condiciones de accesibilidad para la población, lo cual mejorara la calidad de la educación.
\end{abstract}

Palabras clave: accesibilidad; planeamiento regional; redes de transporte, calidad de la educación

\section{Quality in Education and Transport Infrastructure from the Territorial Accessibility Pont of View. Caldas Department in Colombia as a Study Case}

\begin{abstract}
In this research, the current relation between the operative characteristics supplied by the transport infrastructure network of the department of Caldas (Colombia) and the results obtained in the Saber 11 test by educational institutions during 2015 is stablished. This test is the one considered by higher education institutions for admission of new students. Geographic coverage for all the Caldas' schools is calculated based on the transport infrastructure network and the results are analysed according to the grade obtained in the test for each institution. The final results allowed stablishing the direct relation between educational quality supplied and the territorial accessibility conditions to the educational institutions in the department, in terms of average travel time invested. The research identifies the relevance of having educational institutions with high accessibility conditions for the community, which would enhance the quality of education.
\end{abstract}

Keywords: accessibility; regional planning; transport network; quality education 


\section{INTRODUCCIÓN}

El Gobierno Nacional, a través de los Planes de Desarrollo, ha buscado establecer una educación de calidad y accesible para la comunidad, en búsqueda de formar mejores seres humanos, más competentes para un mercado altamente calificado, ciudadanos que aporten al desarrollo el país a través de la ética y las buenas practicas, ejerciendo sus derechos y cumpliendo los deberes. El objetivo de Colombia es convertirse en el país más educado de América Latina para el año 2025 (Ministerio de Educación, 2016). La planificación territorial subyace en la aplicación de políticas públicas que no sólo busquen el proveer a la población las mejores alternativas de desarrollo en sí, sino que permitan la aplicación de instrumentos cuantitativos, que a través de relaciones geoespaciales, vislumbren las mejores alternativas de ubicación de variados nodos de actividad o equipamientos. El proveer un mejor y adecuado acceso a servicios considerados de primera necesidad, hace parte de las metas en transporte de una amplia cantidad de países en el mundo (Jones, 2011), enfilando esfuerzos hacia la reducción de diferencias entre clases sociales. La educación es un servicio que debe ser asequible, accesible y de excelente calidad, convirtiéndole en un artículo de primera necesidad para una comunidad.

El Departamento de Caldas se ubica en la región centro occidente de Colombia, sobre la cordillera central (Ver Figura 1), registrando altitudes que van desde los 200 hasta los 5,432 msnm; se encuentra localizado entre los $5^{\circ} 46^{\prime} 51^{\prime \prime}$ y los $4^{\circ} 48^{\prime} 20^{\prime \prime}$ latitud norte con $74^{\circ} 38^{\prime} 01^{\prime \prime}$ y $75^{\circ} 55^{\prime} 45^{\prime \prime}$ longitud oeste. En Caldas se asientan 987,991 habitantes (2.1\% de la población nacional) en los 27 municipios que le componen, ocupando una superficie de 789 mil Ha representado el 0.69\% del territorio nacional (Gobernación de Caldas et al., 2016).

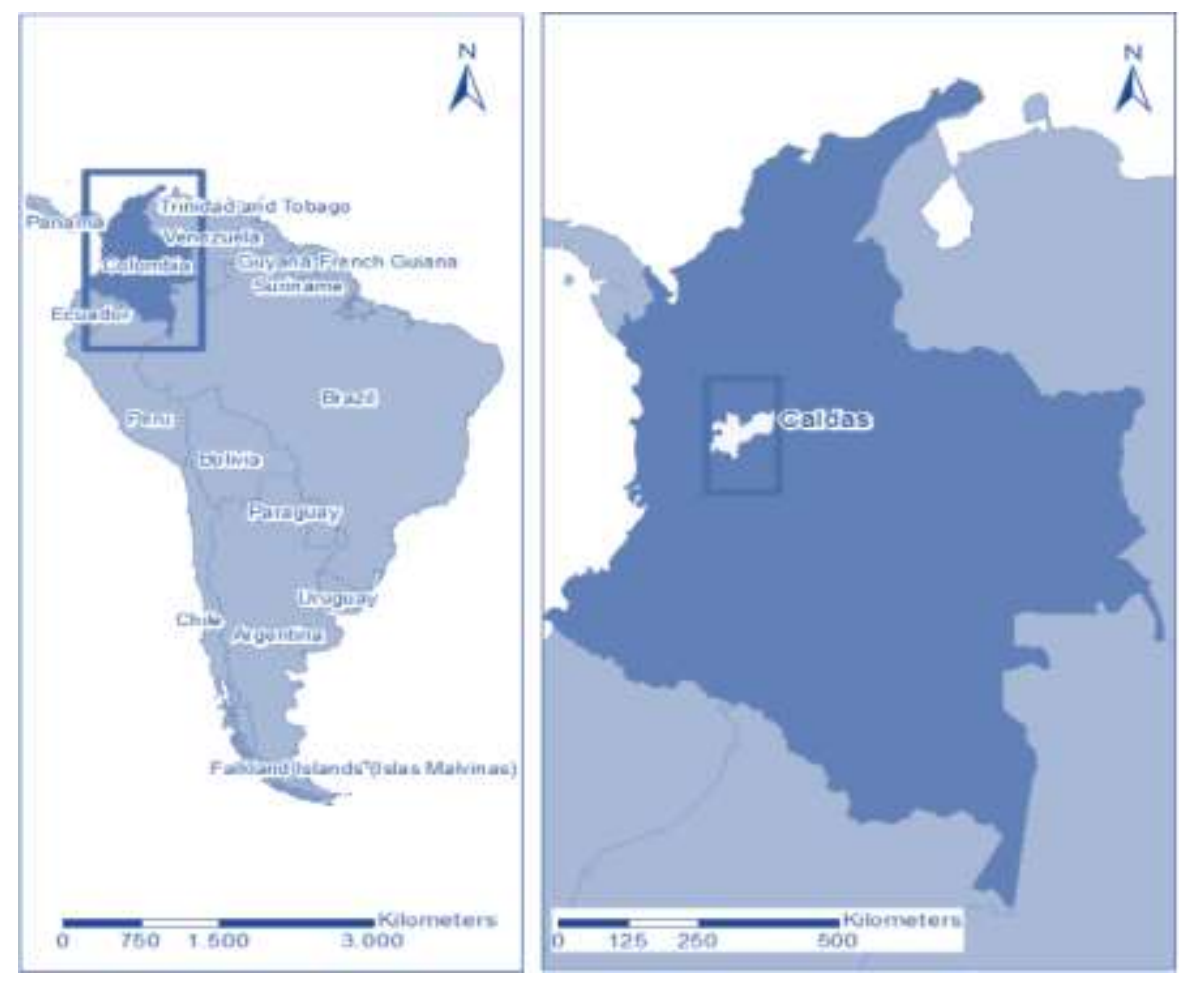

Fig. 1: Ubicación geográfica del Departamento de Caldas.

La brecha en el logro educativo, entendida como como la diferencia de la calidad educativa entre planteles públicos y privados, para los municipios que conforman el Departamento de Caldas, es superior al $70 \%$, excepto para su capital Manizales (Escobar et al., 2016), en donde se ubican la mayor cantidad de Instituciones de Educación Superior en el Departamento. Por otra parte, Colombia ha reducido de una manera importante la tasa de analfabetismos; paso de un 13,5\% en 1985 a 7,6\% para el año 2003, mientras Caldas paso de un 8,9 a 7,3 en el mismo periodo de Tiempo (DNP et al., 2003), sin embargo clasificando los porcentajes en rangos de edades, los jóvenes caldenses de 15 a 24 años muestran mayor porcentaje de analfabetismo que el conglomerado Nacional. En relación con las tasas de cobertura neta, ésta ha disminuido del $83 \%$ al 77\% entre los años 2008 y 2015, encontrando que el caso más crítico de disminución de cobertura se presentan en el nivel educativo de Primaria (disminución del 19\%), mientras que los niveles de educación media y básica, que son los niveles anteriores al nivel de educación superior, han aumentado su cobertura en un $4 \%$ y un $6 \%$, respectivamente (Gobernación de Caldas, 2016; Escobar et al., 2016). Lo anterior muestra que es la población joven la que actualmente se enfrenta problemas de cobertura educativa, así mismo, muestra que el educación superior debe estar preparada para ofrecer cada vez más cupos a estudiantes que finalicen su educación media y básica. 
La educación ha sido identificada como el factor más importante para la formación humana de un país como medida de desarrollo (Hanmer, 1998). Para Colombia, este aspecto juega un rol fundamental en búsqueda de desarrollo, y más cuando actualmente existe un proceso de paz que busca disminuir la violencia, factor que ha influenciado negativamente en el desarrollo económico y social de los ciudadanos; por tal motivo, la localización optima de los equipamientos se constituye un factor determinante para el bienestar social, apoyo al desarrollo, estructura interna de los asentamiento humanos (Garnica, 2012). Para brindar educación de calidad, se debe desarrollar la eficiencia espacial, minimizando los tiempos promedios de viaje desde los hogares hacia los colegios (Bosque y Moreno, 2004).

En Colombia, en términos de calidad de la educación básica y media, se utilizan índices de medición a partir de pruebas escritas en los años $3^{\circ}$ y $5^{\circ}$ (educación básica primaria), año $9^{\circ}$ (educación básica secundaria) y año $11^{\circ}$ (educación media); para el año 2015, Caldas obtuvo (en una escala de 1 a 10) 4,8; 4,49 y 4,99 para la Educación básica primaria, básica secundaria y media respectivamente; para el año 2016, el Departamento mejoro el puntaje obteniendo 5,39; 4,94 y 5,38; sin embargo siguen por debajo de la media Nacional $(5,42$ básica primaria; 5,27 básica secundaria y 5,89 media) (La Patria, 2016). Las pruebas SABER 11 se aplican a todos los estudiantes que han finalizado su formación media, siendo una prueba de obligatoria presentación no sólo para graduarse como bachilleres, sino para presentarse a los procesos de admisión a la educación superior; se escogen los resultados de estas pruebas para la presente investigación, entendiendo la alta importancia de las mismas para los estudiantes, ya que marca claramente un antes y un después en su desarrollo como personas y define las posibilidades de formación como futuros técnicos o profesionales. Respecto a los resultados históricos de las Pruebas Saber 11 (Ver Figura 2); a pesar de ser valores que resaltan un déficit de calidad de educación (35\% nivel bajo, $44 \%$ nivel medio y $21 \%$ nivel alto para el año 2011), el Departamento de Caldas sobresale a nivel Nacional y en el Eje Cafetero, obteniendo mejores resultados generales.

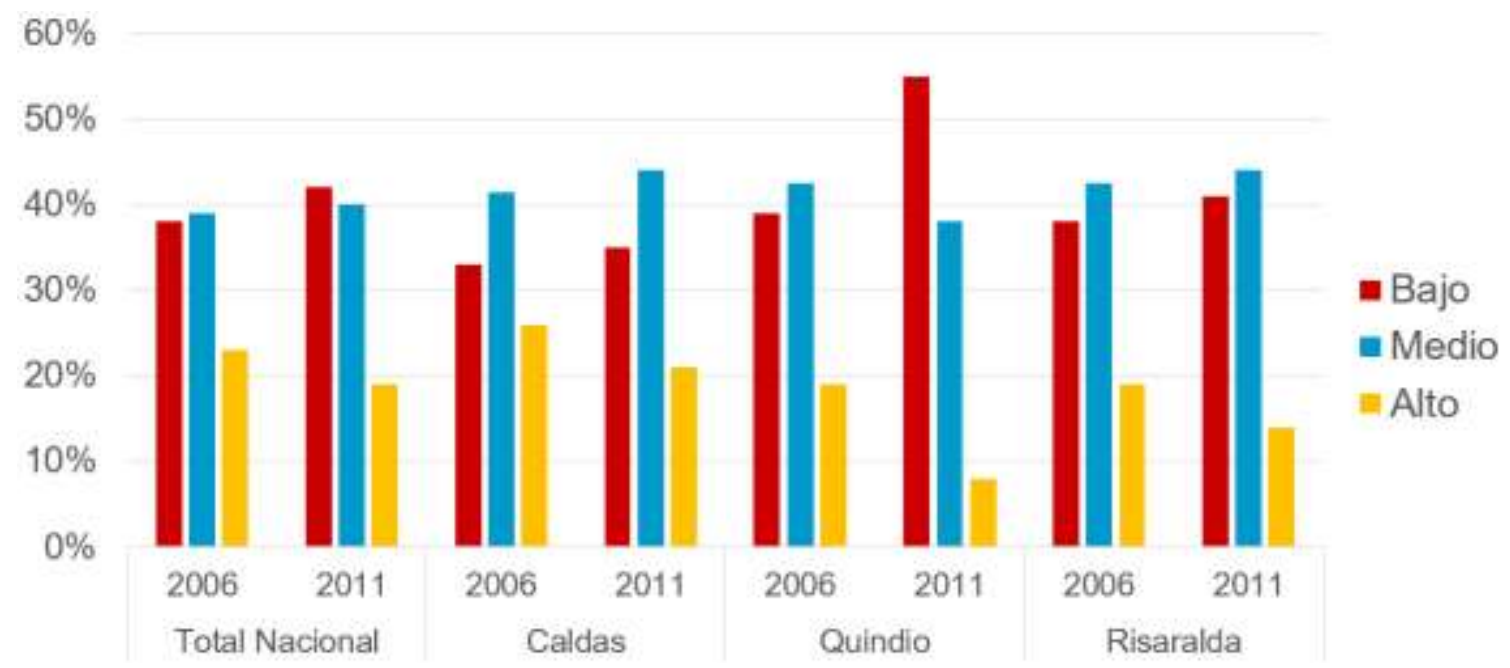

Fig. 2: Clasificación Pruebas Saber 11 de los estudiantes nuevos que ingresan a la educación superior. Fuente: SPADIES en Ministerio de Educación, Informe de Diagnostico de deserción en los Departamentos de Caldas, Quindío y Risaralda, 2012.

La presente investigación enfoca sus esfuerzos en realizar un análisis de las características de accesibilidad media global que las infraestructuras del transporte ofrecen a los centros educativos del Departamento de caldas para luego ser comparada con los puntajes obtenidos en la prueba SABER 11. Se busca establecer la relación existente entre la ubicación geoespacial de dichos equipamientos y las características operativas de la red vial, a través de la aplicación de técnicas geo estadísticas.

Luego de la introducción se aborda una revisión literaria concreta, posteriormente se presenta la metodología aplicada en la investigación, los principales resultados obtenidos y discusión al respecto; finalmente se mencionan las principales conclusiones de la investigación y el trabajo futuro que puede desprenderse de esta investigación.

\section{REVISIÓN DE LA LITERATURA}

La primera definición técnica del concepto "accesibilidad" fue expresada por Hansen (1959) "the potencial of opportunities for interaction", a pesar de ello, el concepto se comenzó a usar en la segunda década del siglo pasado en investigaciones relacionadas con la teoría de la localización y el planeamiento económico regional 
(Batty, 2009); Garrocho y Campos (2006), proponen dos clases de accesibilidad, la potencial, relacionada con la probabilidad de contacto e interacción, coincidiendo con Martínez (2012), y la accesibilidad real, que se relaciona con el uso comprobable del servicio. Los análisis de accesibilidad permiten comprender el impacto que sobre las actividades humanas, como por ejemplo la educación, tienen las inversiones en infraestructura del transporte y en general el impacto que tiene la economía sobre dichas actividades (Geurs y Van Wee, 2004). Por otra parte, en la última década se han mejorado las herramientas informáticas que comúnmente se usan para realizar análisis espaciales mediante la agregación y relación de diferentes bases de datos de índole geográfico (Zhu y Liu, 2004), estas herramientas han hecho posible que los investigadores puedan integrar información geográfica y espacial de los nodos de actividad e información relacionada con características sociodemográficas y socioeconómicas de las regiones y características de las redes de infraestructuras de transporte que por dichas regiones existe disponible; es así como las infraestructuras del transporte se involucran en el análisis, y por ende, en la planificación del contexto regional.

En la búsqueda de una adecuada planificación territorial es importante identificar los sectores de una región que tienen menos probabilidades o posibilidades de alcanzar cierto tipo de actividad o servicio, lo cual se puede realizar mediante un análisis e accesibilidad, el cual, a partir de la teoría de grafos (Segui y Petrus, 1991), aplica un análisis morfométrico de redes que explicaría el aspecto que posee la estructura completa de una red. El concepto se relaciona con la variable distancia, y más directamente con la distancia a las infraestructuras de transporte y la manera como dichas infraestructuras disminuye los tiempos medios de viaje entre dos puntos específicos (Morris et al., 1978). Autores como Biehl (1986) y Gutiérrez et al., (1994), han definido cuatro variables determinantes del desarrollo regional: las infraestructuras, la localización de actividades, la aglomeración y estructura de asentamientos y, finalmente, la estructura económica sectorial; expresando entonces que las infraestructuras de transporte se convierten en un elemento importante en la aplicación de políticas de desarrollo territorial (Gutiérrez et al., 1994; Escobar et al., 2013). La accesibilidad a un territorio es una medida del factor de competitividad de una región (Biehl, 1991), estableciéndose que las poblaciones con condiciones de accesibilidad más adecuadas han reportado una mayor evolución económica, comprobándose que el mejoramiento de las infraestructuras de transporte es una variable clave en el desarrollo económico (Holl, 2007). Un análisis de accesibilidad se configura entonces como un apoyo técnico que puede soportar la toma de decisiones relacionadas con intervenciones que se pretendan realizar a la red de infraestructuras y máxime para definir en qué zonas de la región se deben hacer esfuerzos para mejorar las condiciones de accesibilidad y calidad de vida de la comunidad (Escobar y García, 2012).

Es posible encontrar en la literatura variados tipos de análisis de accesibilidad, abordando temas como: operatividad de modos de transporte (Escobar et al., 2015); cohesión social (Schürmann et al., 1997); crecimiento urbano, densidad poblacional y plusvalía (Alonso, 1964); análisis geoespacial de actividades económicas (Krugman, 1991); marketing (Geurs y van Eck, 2001), planes de evacuación (Padilla et al., 2016); Prestación y localización de servicios (Calcuttawala, 2006); sostenibilidad (Escobar et al., 2013); exclusión social (Preston y Rajé, 2007); entre otros campos.

Es claro entonces que los análisis de accesibilidad permiten conocer las posibilidades de interacción entre la ubicación de servicios o nodos de actividad y la comunidad en general, mediante el uso de las infraestructuras del transporte, ayudando a identificar las áreas de la región que no se encuentran servidas por un centro educativo (Jones y Kauffman, 1994), o analizando el impacto de la accesibilidad geográfica en el sistema educativo (Sá et al., 2005); situación que se debe considerar al definirse la aplicación de modelos territoriales regionales con conceptos sustentables y equitativos (Venegas y Rojas, 2009). Ahora bien, también se han realizado estudios en los cuales se investigan los factores determinantes de la entrada a la universidad, en términos tanto de su accesibilidad geográfica como de la calidad de enseñanza de los programas ofertados (Sá et al., 2003); así como estudios en donde los logros estudiantiles mostraron que la distancia a la escuela tuvo un efecto significativo e inversamente relacionado con las calificaciones obtenidas por los estudiantes de tercer grado (Talen, 2001). No obstante, la accesibilidad ha sido un instrumento de planificación territorial y diagnóstico poco usado en Colombia y Latinoamérica, sin embargo, la aplicación de este concepto es tan importante que debe comprenderse como una necesidad secundaria (Halden, 2011) no percibida.

Por lo tanto, los modelos de accesibilidad, son una herramienta importante para el planeamiento del territorio, en el cual se busca impulsar el potencial, económico y social de un país, departamento, ciudad o región. La educación es sin duda, uno de los pilares más importantes para el desarrollo de los estados; no solo influye en el desarrollo de profesionales que brindaran mejoras en la economía, sino también en el impulso social, aumentando la cohesión social, disminuyendo la inequidad, mejorando la calidad de vida de las personas, entre muchos otros aspectos.

\section{METODOLOGÍA}

La metodología de investigación se basa en la ejecución de cinco (5) etapas consecutivas, cuya secuencia se presenta en la Figura 3 y las cuales, de forma general se definen como sigue: 


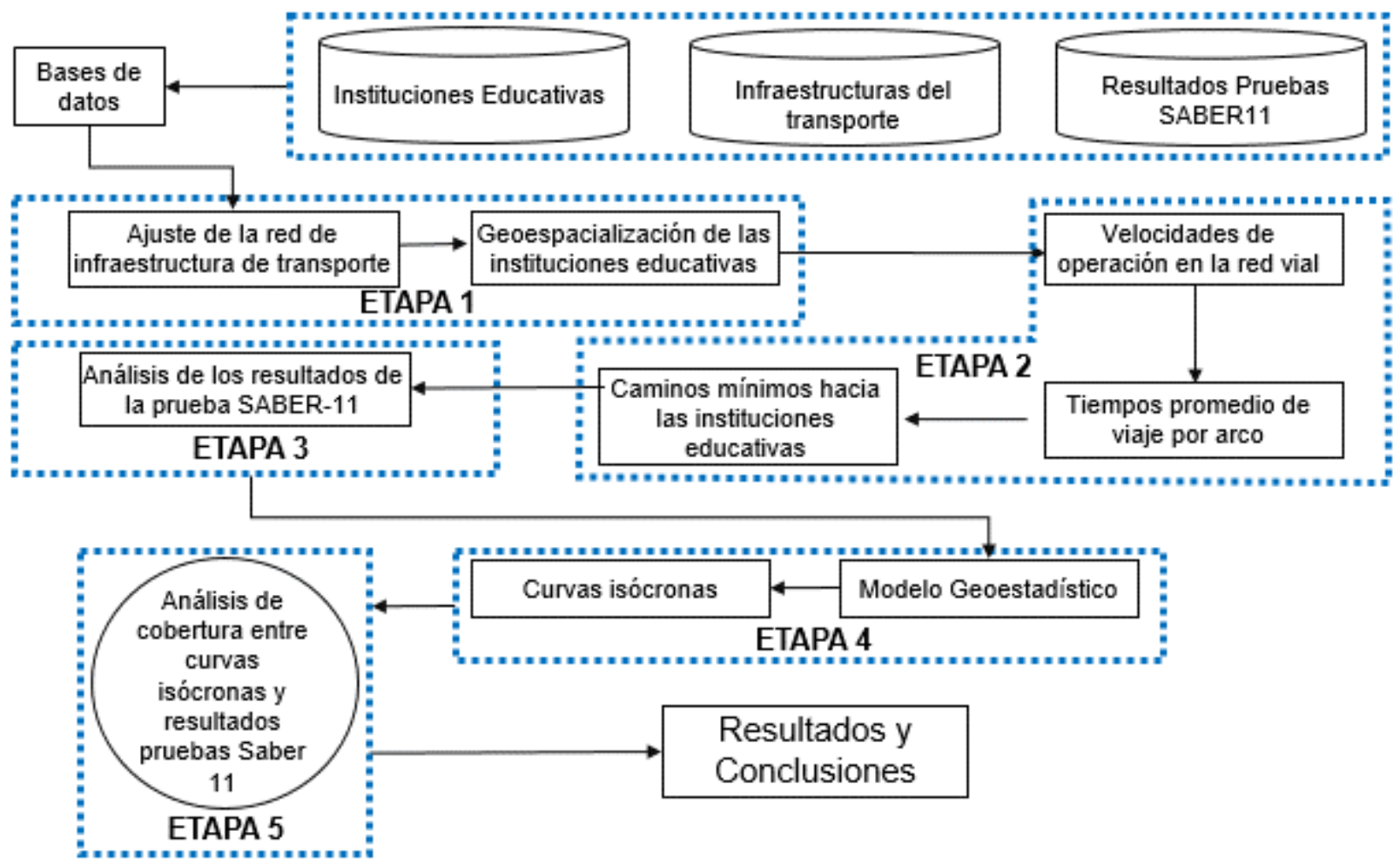

Fig. 3: Metodología abordada en la investigación.

Etapa 1, Ajuste de la red de infraestructuras de transporte. Esta etapa se relacionad directamente con el ajuste de la red georeferenciada a través de la adquisición de información actualizada sobre la red vial. Se consultó información secundaria que fue obtenida en la Secretaría de Educación, la Secretaría de Infraestructura y la Secretaría de Planeación, las cuales poseen información de primera mano sobre la ubicación geoespacial de las instituciones educativas que se encuentran en el Departamento de Caldas, e información respecto a la infraestructura del transporte que actualmente ofrece el Departamento. El Plan Vial Regional del año 2009 (Gobernación de Caldas, 2009) fue el punto de partida en dicha pesquisa, para la verificación de los datos se realizaron salidas de campo, y apoyados con equipos GPS fue posible rectificar el trazado vial y la ubicación de las instituciones educativas con mayor precisión. Simultáneamente, se obtuvo la base de datos de los resultados de las pruebas Saber 11 del año 2015 para todas las instituciones del Departamento Caldense. Con la anterior información se consolidó la base de datos a ser usada para el modelo de accesibilidad y análisis geoestadístico.

Etapa 2, cálculo de las velocidades de operación promedio de los arcos. Esta etapa se basó en el complemento de información de las características operativas de la red vial a través del uso de dispositivos GPS, y en base a datos obtenidos en investigaciones anteriores realizadas en la región Centro-Sur del Departamento de Caldas (Escobar et al., 2013).

Etapa 3, análisis de los resultados de la prueba Saber 11 del año 2015. Se realizó un análisis ponderado por municipios y por subregiones aplicando la Expresión (1), en donde: i= Instituciones educativas del Departamento de Caldas; $R_{s 11 i}=$ Resultado en la prueba Saber 11 para el colegio i; $\mathrm{y} m_{i}=$ número de personas evaluadas para el colegio i.

$$
\frac{\sum_{i=1}^{n} R_{s 11 i} * m_{i}}{\sum_{i=1}^{n} m_{i}} \quad i=1,2,3, \ldots, n
$$

Etapa 4, cálculo de la Accesibilidad Media Global. Esta etapa se analizó a partir del vector de tiempo medio de viaje $\left(T_{v i}\right)$, el cual representa el tiempo promedio de viaje desde el nodo i hasta los demás nodos de la red. Para el cálculo, se utilizó un algoritmo del SIG que permite calcular la menor impedancia (camino mínimo) entre un nodo específico y los demás nodos de la red, generándose la matriz de impedancias. A través de esta matriz y en base a la velocidad de operación promedio de cada arco, se elaboró la matriz de tiempos promedios mínimos de viaje, en la que se minimiza el tiempo medio de viaje entre todos y cada uno de los nodos que conforman la red en estudio. Una vez determinada la matriz de tiempos promedios mínimos de viaje, se obtuvo el vector de tiempo promedio de viaje $\left(T_{v i}\right.$, Expresión (2)). Donde, $T_{v i}=$ tiempo de viaje mínimo promedio entre el nodo i y los demás nodos de la red; $n=$ número de nodos de la red. 


$$
\overline{T_{v \imath}}=\frac{\sum_{j=1}^{n} t_{v i}}{(n-1)} \quad i=1,2,3, \ldots, n ; j=1,2,3, \ldots, \mathrm{n}
$$

El vector de tiempo medio de viaje obtenido ( $\mathrm{n} \times 1$ ), se relaciona con las coordenadas geográficas (longitud y latitud) de cada uno de los nodos, obteniéndose así la matriz de orden ( $n \times 3)$, a través de la cual se generan las curvas isócronas de tiempo promedio de viaje para el análisis de la Accesibilidad Media Global. Para los cálculos geoestadísticos se usó el Método de Kriging ordinario con semivariograma lineal como modelo de predicción de los tiempos medios de viaje. El semivariograma caracteriza las propiedades de dependencia espacial entre puntos pertenecientes a una muestra observada, que se calcula mediante la Expresión (3), donde $Z_{(x)}=$ valor de la variable en un sitio con coordenadas $\mathrm{x}, \mathrm{y} ; Z_{(x+h)}=$ otro valor muestral separado por una distancia $\mathrm{h} ; \mathrm{n}=$ número de parejas que se encuentran separadas por dicha distancia. Se resalta que a menor distancia, mayor la similitud o correlación espacial entre las observaciones. El método Kriging ordinario propone que el valor de la variable puede predecirse como una combinación lineal de las $\mathrm{n}$ variables aleatorias, como se presenta en la Expresión (4), en donde $\lambda_{i}=$ los pesos o ponderaciones de los valores originales.

$$
\begin{aligned}
& \overline{\gamma_{(h)}}=\frac{\sum\left(Z_{(x+h)}-Z_{(x)}\right)^{2}}{2 n} \\
& Z_{(x 0)}=\lambda_{1} Z_{(x 1)}+\lambda_{2} Z_{(x 2)}+\lambda_{3} Z_{(x 3)}+\lambda_{4} Z_{(x 4)}+. .+\lambda_{n} Z_{(x n)}=\sum_{i=1}^{n} \lambda_{i} Z_{(x i)}
\end{aligned}
$$

Etapa 5, análisis de coberturas. Se basa en establecer la relación existente entre los resultados de las pruebas Saber 11 ponderado por las personas evaluadas y por el área de los municipios para cada una de las curvas de accesibilidad media global, a partir de análisis de cobertura geoespacial. Se generan planos que permiten visualizar gráficamente las principales conclusiones de la investigación.

\section{RESULTADOS Y DISCUSIÓN}

Después de analizar la base de datos de los resultados de las pruebas Saber 11 de cada colegio para el año 2015, se procede a evaluar los resultados por municipio. Manizales se destaca por ser el municipio del Departamento de Caldas con mayor número de alumnos evaluados alcanzando valores de 3500 estudiantes en colegios oficiales y más de 1000 estudiantes de colegios privados; mientras que el municipio de Marulanda registra tan sólo 34 estudiantes de colegios oficiales que encararon las pruebas Saber 11 en el 2015 . En la Figura 3, se observa la clasificación de los resultados de las pruebas Saber 11 obtenida para cada municipio, ponderado por el número de estudiantes, en colegios oficiales del departamento; se observa que los municipios de Neira, Manizales y Villamaría son los que registran mejores calificaciones; esto se explica por el hecho de que sea la zona de más desarrollo económico, social y alta urbanización, debido a la influencia de Manizales, la Capital del Departamento. Se resalta que el municipio de Neira (sombreado verde) obtenga un resultado ponderado mayor que Manizales, mostrando mejores calidades en términos educativos, coincidiendo con ser el municipio de la región centro-sur con mayor índice de instituciones educativas por cada 100 mil habitantes, 177 (Escobar et al., 2016).

Así mismo, se observa que municipios en los cuales la forma de ocupación preponderante es la rural, los resultados de las pruebas SABER11 son bajos; como ejemplo de ello se encuentra precisamente el municipio de Marulanda (sombreado rojo en Figura 4), cuyas características de ocupación son predominantemente rurales y las posibilidades de acceder al servicio educativo son más limitadas que en otros municipios con características de ocupación más urbana o de alta densidad como el municipio de Neira o de Manizales (Sombreados en verde en Figura 4).

En la Figura 5, se presenta la clasificación de los resultados de las pruebas Saber 11 obtenida para cada municipio, ponderado por el número de estudiantes, en colegios privados del departamento; se observa que Manizales registra los mejores puntajes, seguido de Anserma y Villamaría; mientras que Palestina es el de puntajes más bajos de los colegios privados. Se destaca que los municipios donde existen colegios privados son Manizales, Anserma, Chinchiná, la Dorada, Manzanares, Palestina y Villamaría, en el resto de municipios del departamento, sólo existen colegios oficiales. Comparando los valores obtenidos entre colegios privados y oficiales, se tiene que los privados obtienen mayores puntajes ponderados en las pruebas Saber 11 que los oficiales, no obstante, también se encuentran colegios privados con puntajes menores al menor valor ponderado obtenido por los colegios oficiales. 


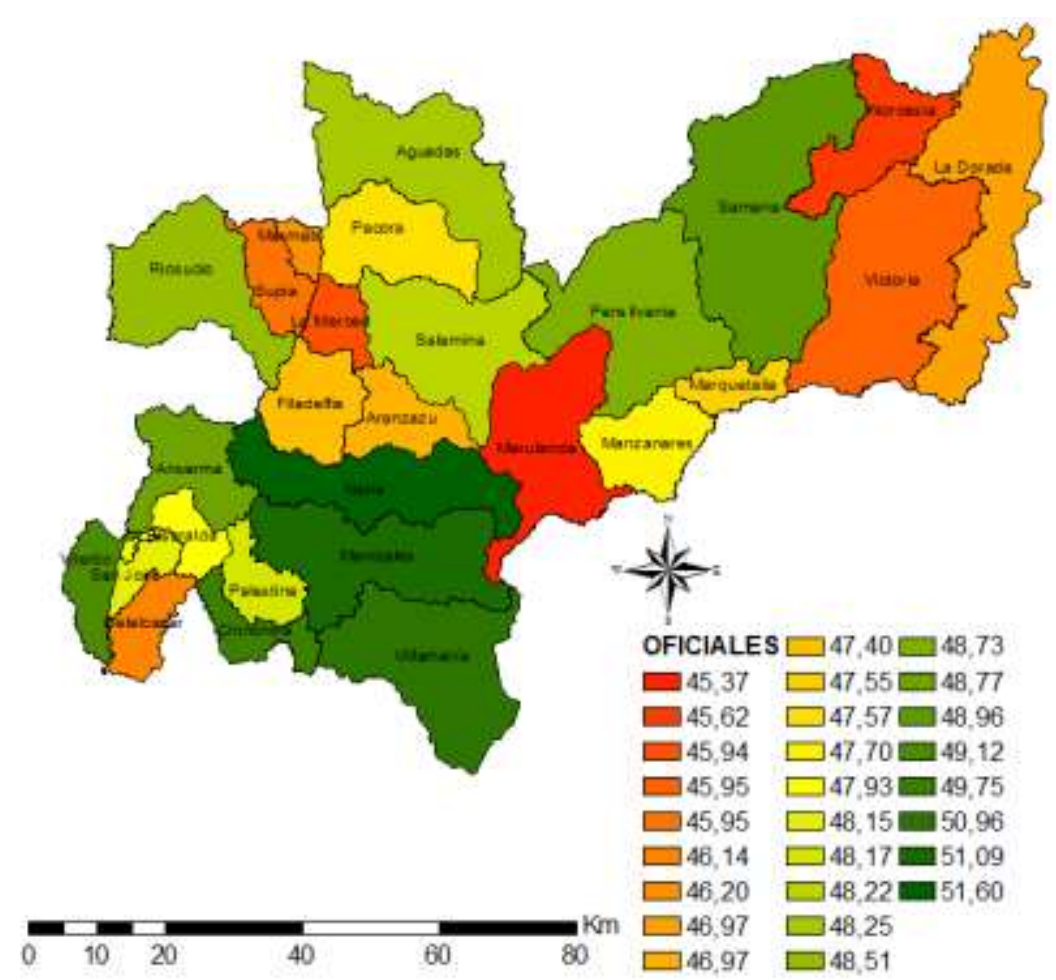

Fig. 4: Resultados de la prueba Saber 11 ponderado por el número de evaluados a nivel municipal. Colegios Oficiales.

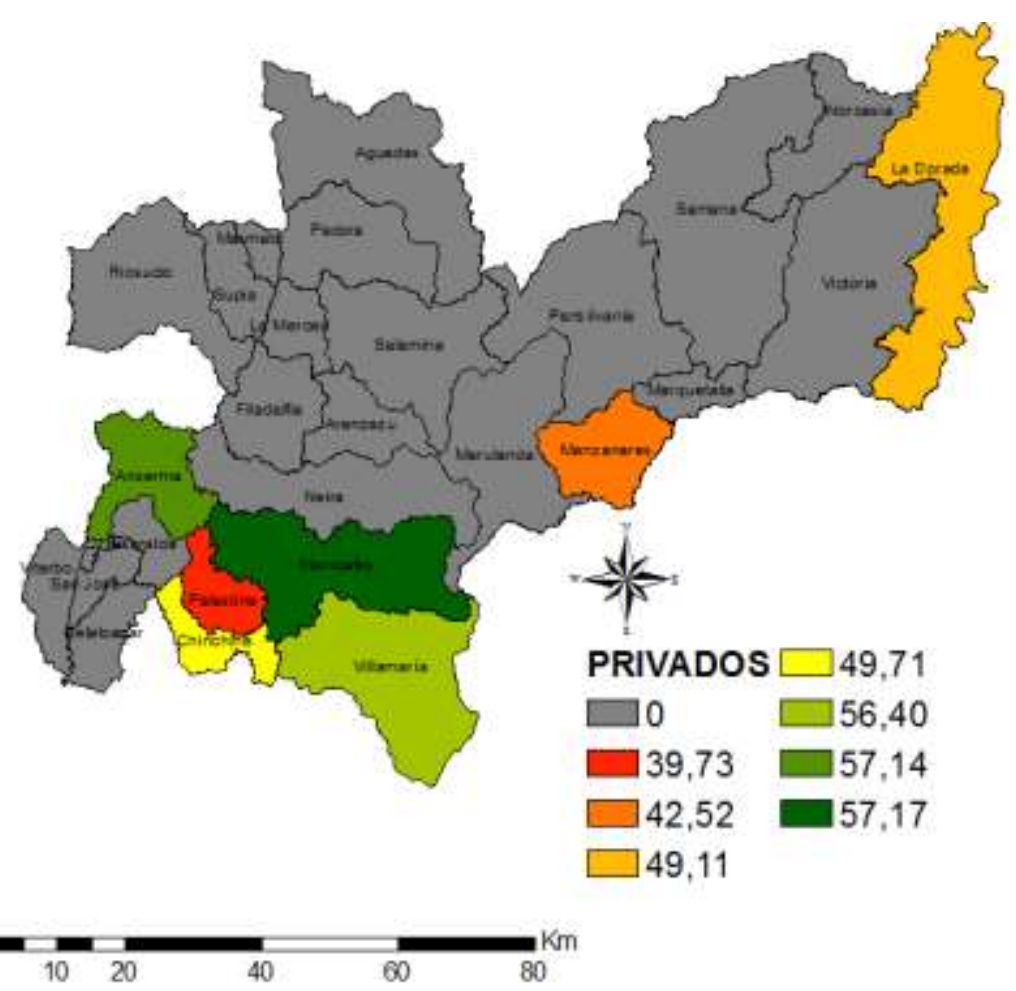

Fig. 5: Resultados de la prueba Saber 11 ponderado por el número de evaluados a nivel municipal. Colegios Privados.

En la Figura 6, se observa la calificación de las pruebas Saber 11, obtenida para cada subregión, ponderado por el número de estudiantes, para colegios oficiales. Las subregiones Centro-Sur y Bajo Occidente registran los mejores puntajes en la prueba; mientras que las subregiones del Oriente y Alto Occidente registran puntajes más bajos. En la Figura 7 se presentan los resultados para los colegios privados, en cada subregión; se observa que la subregión Bajo Occidente es la que mejor puntaje obtuvo en las pruebas Saber 11 en colegios privados, seguido de la subregión Centro-Sur, éstas dos subregiones son las más se destacan en 
términos de las pruebas saber 11 , tanto para colegios privados como oficiales, mientras que el resto de las subregiones del departamento reportan menores resultados en dichas pruebas. A pesar de que las dos subregiones mencionadas, Bajo-Occidente y Centro-Sur son las de mejores resultados en dichas pruebas, son éstas mismas las que refieren un menor número de instituciones educativas por cada 100 mil habitantes, 32 y 18, respectivamente (Escobar et al., 2016).

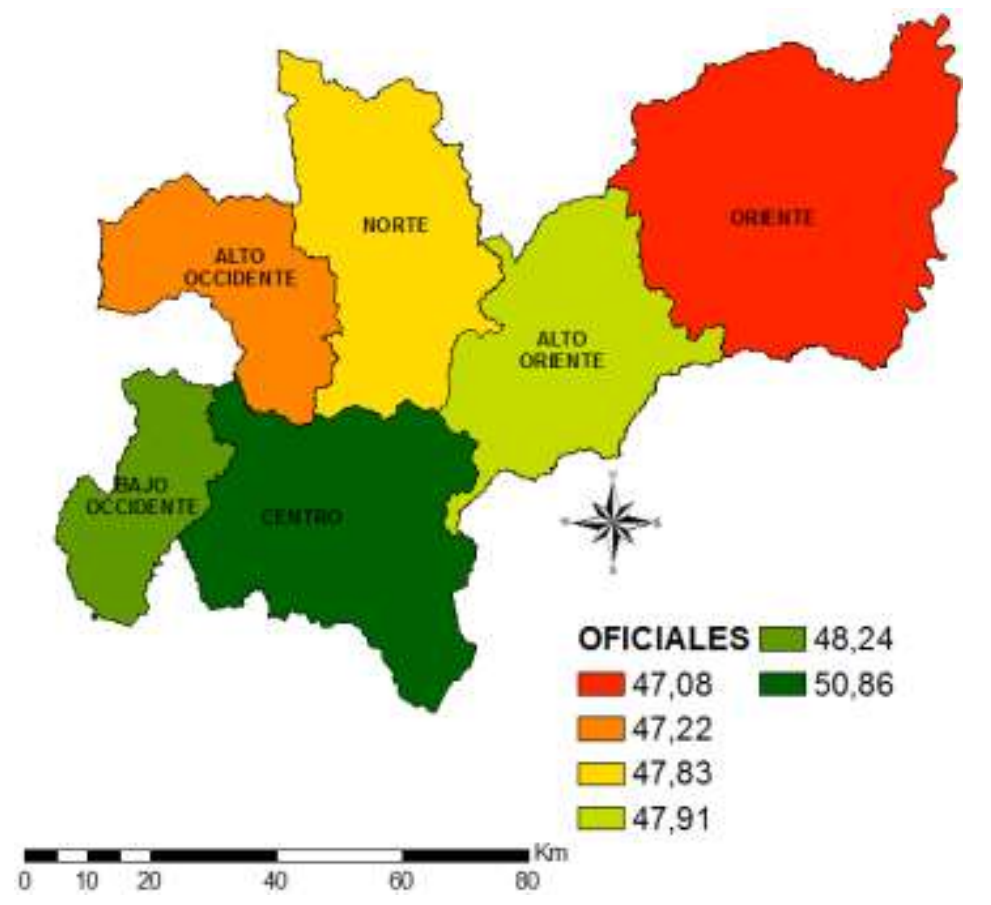

Fig. 6: Resultados de la prueba Saber 11 ponderado por el número de evaluados a nivel subregional. Colegios Oficiales.

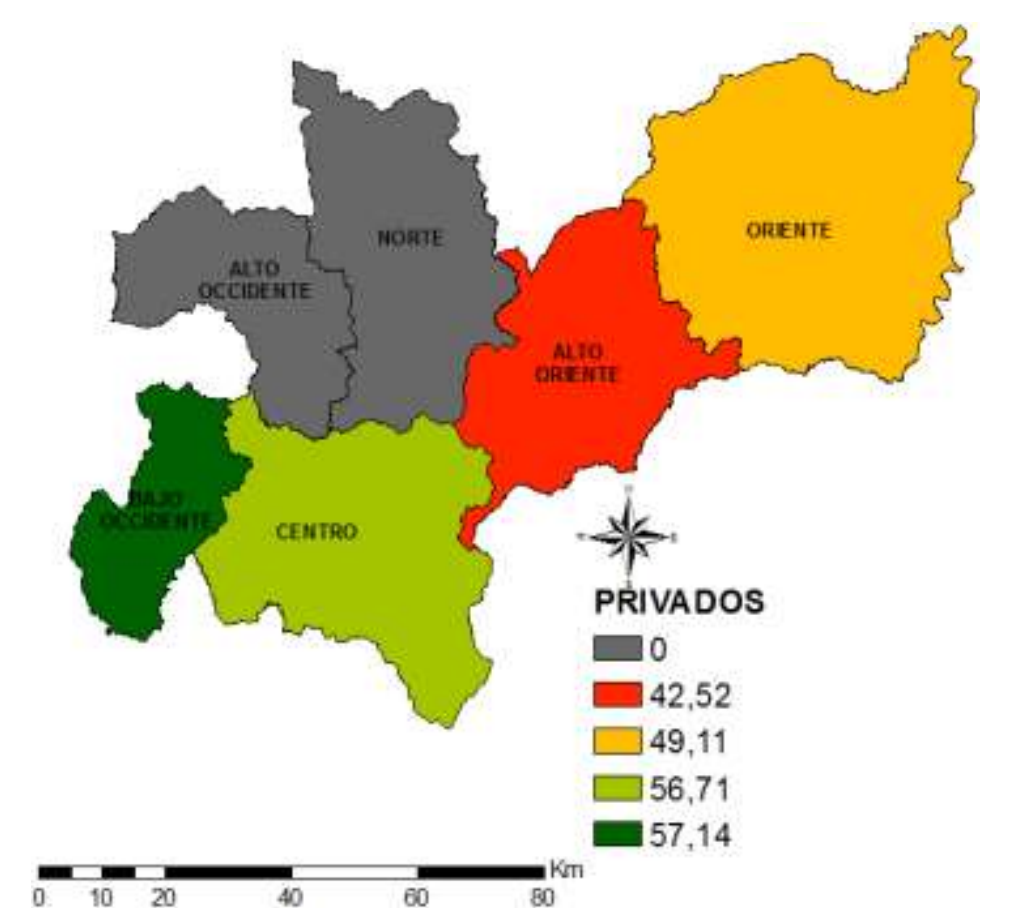

Fig. 7: Resultados de la prueba Saber 11 ponderado por el número de evaluados a nivel subregional. Colegios Privados

En la Figura 8, se observan los resultados del modelo de Accesibilidad Media Global para el Departamento de Caldas. Los municipios de Aránzazu, Filadelfia y Salamina, son los más accesibles con valores aproximados de 130 min en promedio; mientras que los municipios de la Dorada y Norcasia, ambos al oriente, presentan los valores más altos de accesibilidad, que se aproximan hasta 320 minutos en promedio. 


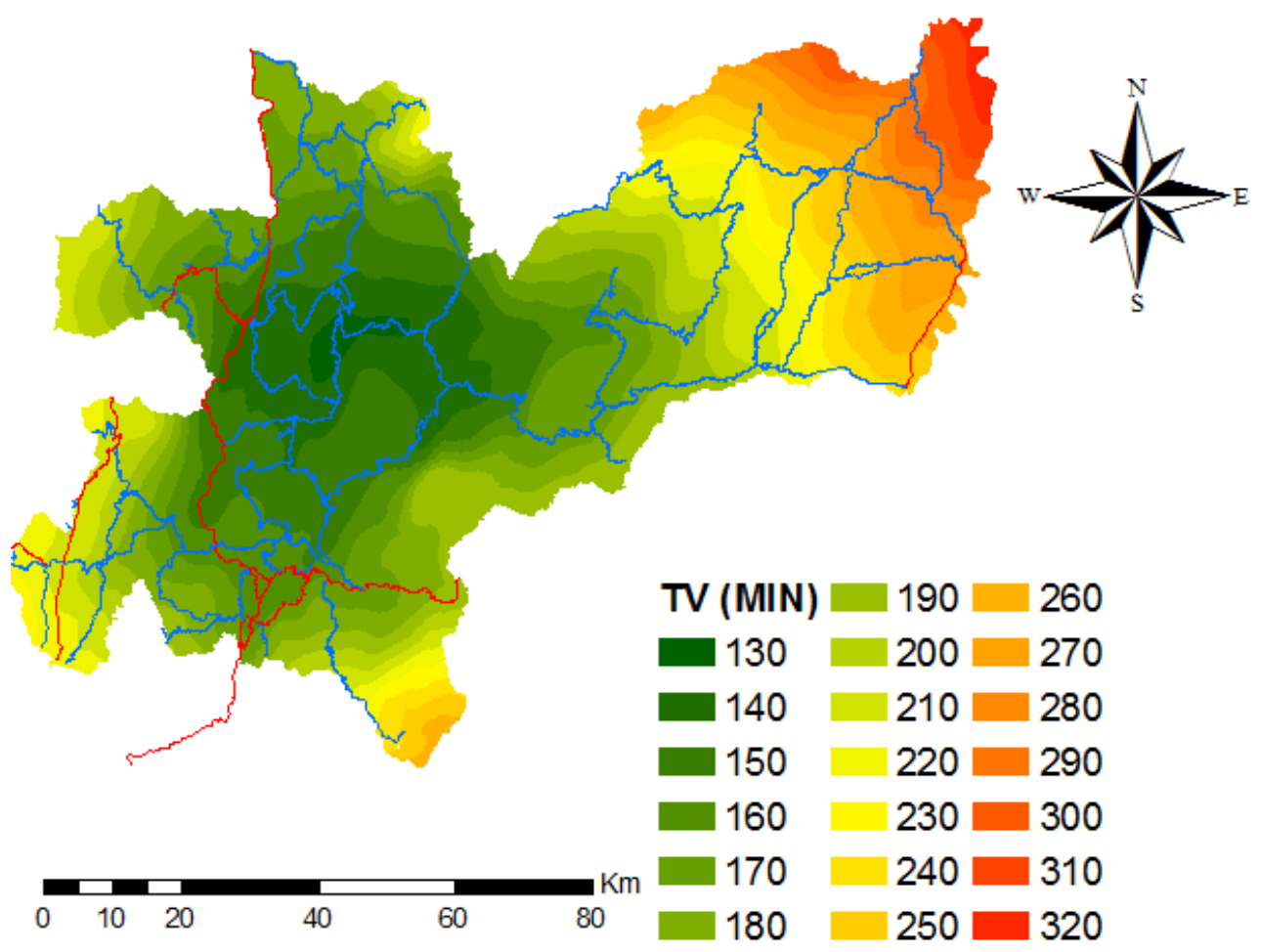

Fig. 8: Accesibilidad Media Global del Departamento de Caldas.

Al analizar la densidad de red vial, se observa que dicha densidad es mayor al occidente del departamento que al oriente. Al realizar el análisis de todas las instituciones educativas del Departamento se obtienen los resultados ponderados por las personas evaluadas, según su nivel de accesibilidad. Por otra parte, en la Figura 9, se presentan los resultados de correlacionar las curvas de accesibilidad media global con los valores obtenidos en la prueba SABER-11.

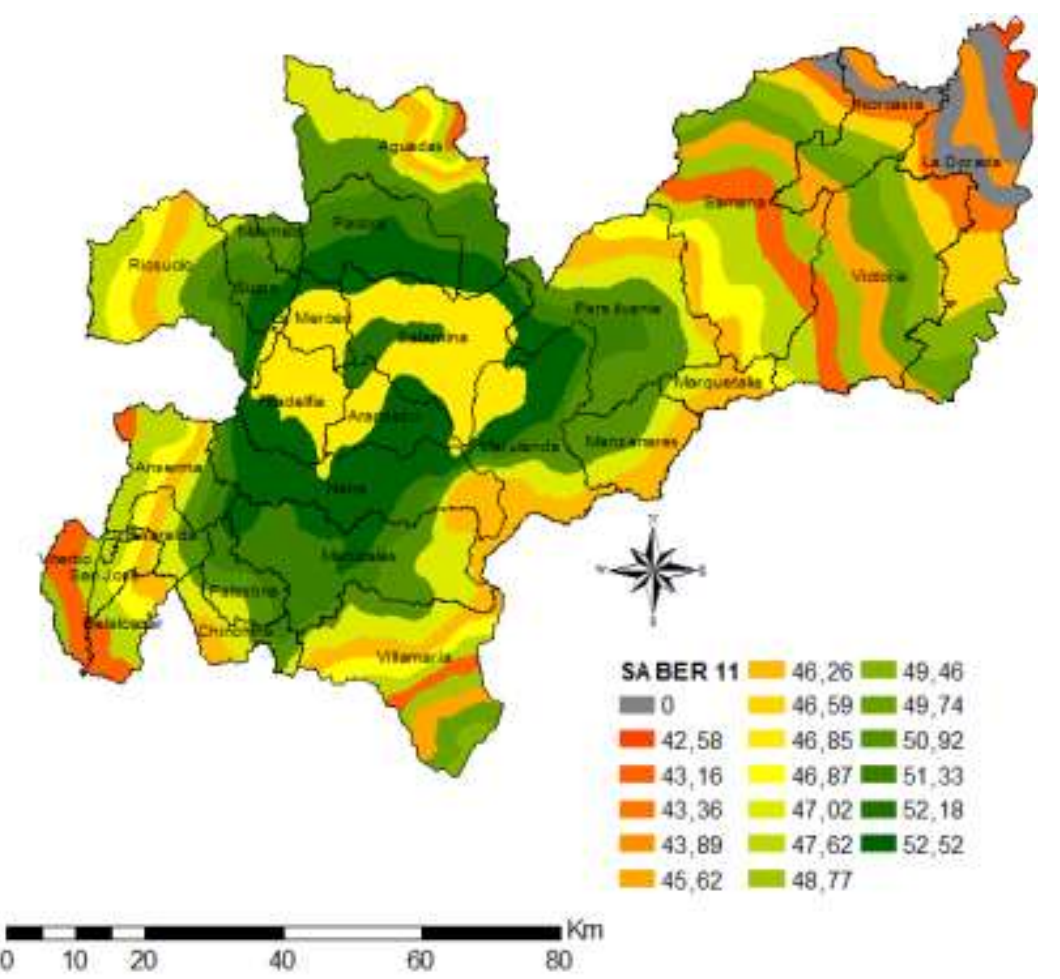

Fig. 9: Resultados de la prueba SABER 11 según el nivel de Accesibilidad.

Se observa que la zona del Bajo Occidente y el Oriente presentan una baja calificación; a pesar de que los resultados obtenidos por el Bajo Occidente son notables, la accesibilidad es deficiente, lo que provoca que obtenga una calificación baja. Del mismo modo al analizar la región Norte, se observa que obtuvo una alta calificación a pesar de los bajos resultados de la prueba SABER-11. Ahora bien, después de obtenidos los 
puntajes de las pruebas Saber 11 para cada una de las curvas isócronas, se realiza una comparación con los límites de cada municipio, buscando establecer cuanto porcentaje pertenece cada curva a cada uno de los 27 municipios. Por tal razón, posterior al cálculo de los resultados ponderados por los estudiantes para cada curva, se calculan los resultados ponderados por el área para cada municipio. En la Figura 10, se observan los resultados; la línea naranja representando el promedio de los resultados ponderados (47,2 puntos), existiendo siete (7) municipios que están por debajo de dicho valor promedio (La Dorada, Norcasia, Samaná, Victoria, Belalcázar, San José y Viterbo).

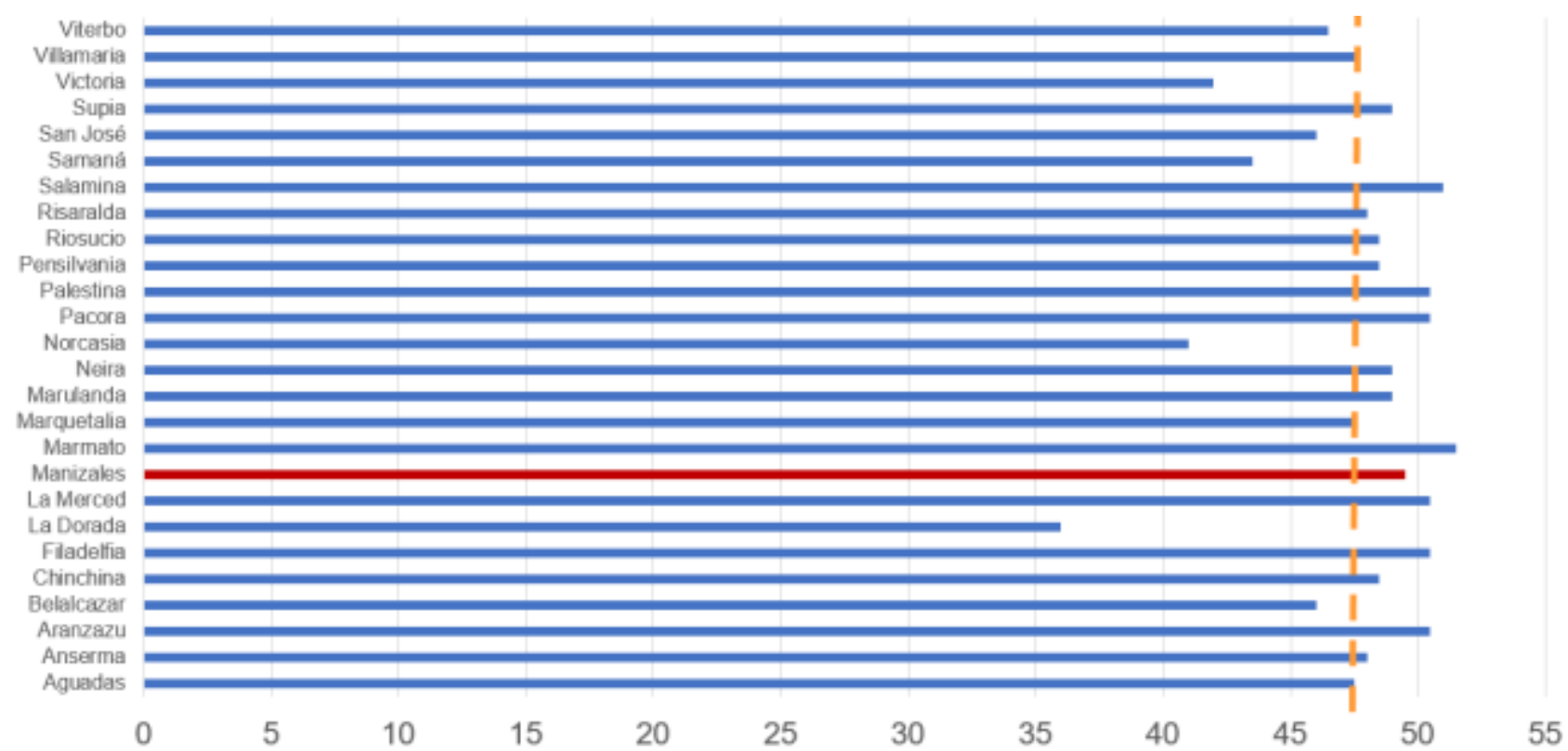

Fig.10: Resultados de la prueba SABER 11 ponderado por el área.

En la Figura 11, se muestra en color verde los municipios que superan el valor medio en los resultados de las pruebas Saber 11; por el contrario en color rojo, se encuentran los municipios con valores por debajo del promedio. Nuevamente, se observa cómo las zonas más accesibles son las que presentan mejores resultados en las pruebas para el acceso a la educación superior.
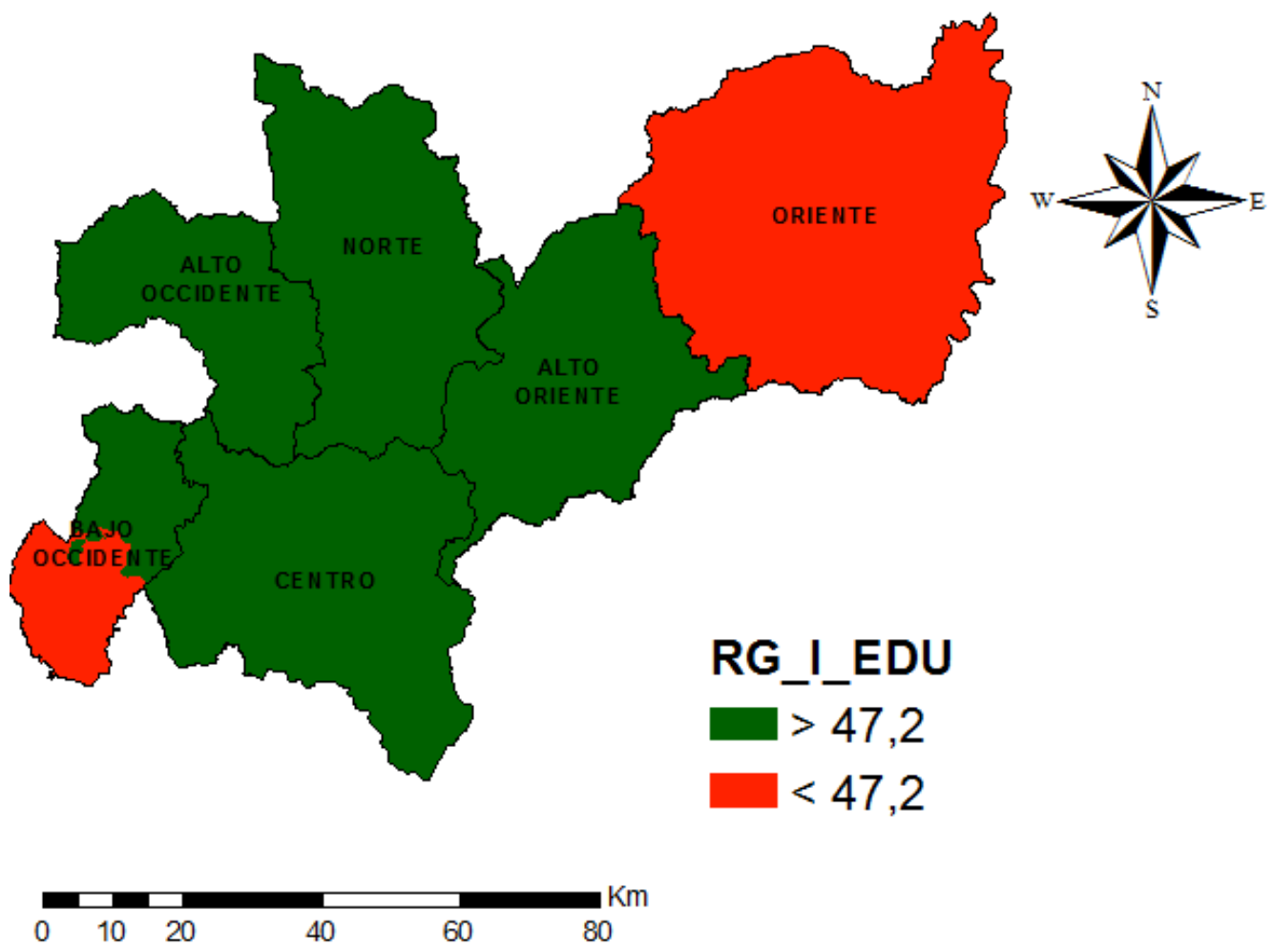

Fig. 11: Municipios del Departamento de Caldas según el promedio de los resultados de las pruebas Saber 11, ponderadas por el área. 


\section{CONCLUSIONES}

Con esta investigación se demostró que no existe una relación entre la oferta en infraestructura vial como acceso a los centros educativos para obtener una educación de calidad. Es de resaltar que las subregiones que poseen unos índices de red vial mayores (Centro, Alto y Bajo Occidente), son precisamente las subregiones que reportan los menores índices de Instituciones Educativas por cada 100 mil habitantes (Escobar et al., 2016), lo cual coincide con los resultados de accesibilidad media global, excepto para la región del bajo occidente.

\section{AGRADECIMIENTOS}

Los autores agradecen al Programa Nacional de Semilleros de Investigación, Creación e Innovación de la Universidad Nacional de Colombia - 2013/2015, ya que a través de éste se obtuvo financiación para la presente investigación con código 23042. Se agradece la colaboración de los estudiantes del Semillero de Investigación en Movilidad Sostenible del Departamento de Ingeniería Civil.

\section{REFERENCIAS}

Alonso, W. Location and land use. Toward a general theory of land rent. Cambridge, MA: Harvard University Press (1964)

Batty, M. Accessibility: in search of a unified theory, doi:10.1068/b3602ed, Environment and Planning B: Planning and Design, 36(2), 191-194 (2009)

Biehl, D. The Contribution of Infrastructure to Regional Development. Final Report. Luxemburgo, Comission of the European Communities (1986)

Biehl, D. The role of infrastructure in regional development. In: Infrastructure and Regional Development, Editorial Pion, 9-35 (1991)

Bosque, J., y Moreno, A. Sistemas de información geográfica y localización de instalaciones y equipamientos. Madrid, Editorial Ra-Ma (2004)

Calcuttawala, Z. Landscapes of information and consumption: A location analysis of public libraries in Calcutta. Edward D. Garten, Delmus E. Williams, James M. Nyce (Ed.), 24, 319-388 (2006)

DNP, UDS, y Misión social. En Plan Sectorial de la Revolución Educativa 2002-2006. Recuperado de: https://goo.gl/hprzFb (2003)

Escobar, D. y García, F. Diagnóstico de la Movilidad Urbana de Manizales, Facultad de Ingeniería y Arquitectura. Universidad Nacional de Colombia. ISBN, 1357143697. Manizales (2012)

Escobar, D., Holguín, J. y Kaffure, C. Análisis de la ubicación geoespacial de las instituciones educativas del Departamento de Caldas (Colombia) y su relación con las características operativas de la red vial. Recuperado de: https://goo.gl/pohXsf Revista Espacios, 37(22), 8 (2016)

Escobar, D., Tapasco, O y Giraldo, J., Medición de Desempeño del Sistema de Transporte Cable Aéreo de la Ciudad de Manizales en Colombia, usando Tres Enfoques: Analítico, Simulado y de Accesibilidad Urbana, doi:10.4067/S0718-07642015000600020. Inf. Tecnol., 26(6), 199-210 (2015)

Escobar D., García F. y Tolosa R. Análisis de Accesibilidad Territorial a Nivel Regional, 1를., Universidad Nacional de Colombia. Facultad de Ingeniería y Arquitectura, Manizales, Colombia (2013)

Garnica, R. Análisis espacial de los equipamientos educativos (oficiales) en la ciudad de Montería, Colombia. Recuperado de: https://goo.gl/h3uYY5. Revista de Geografía, 12, 159-179 (2012)

Garrocho, C. y Campos, J. Un indicador de accesibilidad a unidades de servicios clave para ciudades mexicanas: fundamentos, diseño y aplicación. Recuperado de: https://goo.gl/3SK2mv. Economía, Sociedad y Territorio, 6(61), 1-60 (2006)

Geurs, K. y Ritsema van Eck, J. Accessibility Measures: Review and Applications. RIVM Report 408505006. National Institute of Public Healthand the Environment, Bilthoven (2001)

Geurs, K. y Van Wee, B. Accessibility evaluation of land-use and transport strategies: review and research directions, doi:10.1016/j.jtrangeo.2003.10.005, Journal of Transport Geography, 12(2), 127-140 (2004)

Gobernación de Caldas. Plan Vial de Caldas 2008-2017 (2009) 
Gobernación de Caldas. Informe Educativo del Departamento. Secretaría de Educación Departamental (2016) Gobernación de Caldas, FINAGRO y IGAC. Departamento de Caldas. Recuperado de: https://goo.gl/Lkz6X5 (2016)

Gutiérrez, P., Monzón de Cáceres, A. y Piñero, J. Accesibilidad a los Centros de Actividad Económica en España. Revista de Obras Públicas, 39-49 (1994)

Halden, D., The use and abuse of accessibility measures in UK passenger transport planning, doi:10.1016/j.rtbm.2011.05.001, Transportation Business \& Management, 2, 12-19 (2011)

Hanmer, L. Human capital, targeting and social safety nets: An analysis of household data from Zimbabwe. doi: https://doi.org/10.1080/13600819808424155. Oxford Agrarian Studies, 26(2), 245-265 (1998)

Hansen, W. How accessibility shapes land use, doi:10.1080/01944365908978307, Journal of the American Institute of Planners, 25 (2), 73-76 (1959)

Holl, A. Twenty years of accessibility improvements. The case of the Spanish motorway building programme. doi:10.1016/j.jtrangeo.2006.09.003. Journal of Transport Geography, 15, 286-297. (2007)

Jones, P. Developing and applying interactive visual tools to enhance stakeholder engagement in accessibility planning for mobility disadvantaged groups, doi:10.1016/j.rtbm.2011.08.001, Transportation Business \& Management, 2, 29-41 (2011)

Jones, R. y Kauffman, A. Accessibility to comprehensive higher education in Texas. doi: https://doi.org/10.1016/0362-3319(94)90023-X. The Social Science Journal, 31(3), 263-283 (1994)

Krugman, P. Increasing returns and economic geography. Recuperado de: https://goo.gl/vqFr8V. Journal of Political Economy, 99(3), 483-499 (1991)

La Patria, Caldas mejora, pero sigue rajado en calidad educativa. Recuperado de: https://goo.gl/GWtKuf (2016)

Martínez, H. La accesibilidad regional y el efecto territorial de las estructuras de transporte. Asociación de geógrafos españoles, 59(59), 79-104 (2012)

Ministerio de Educación. Misión, Propósito Superior y Visión. Recuperado de: https://goo.gl/5pZaar. República de Colombia (2016)

Morris, J., Dumble, P. y Wigan, M. Accessibility indicators in transport planning, doi:10.1016/01912607(79)90012-8, Transportation Research, A(13), 91-109 (1979)

Padilla, O., y otros 6 autores. Enhanced vertical evacuation application with geomatic tools for tsunamis in Salinas, Ecuador. Science of Tsunami Hazards, 35(3), 189-213 (2016)

Preston, J. y Rajé, F. Accessibility, mobility and transport-related social exclusion. doi: doi:10.1016/j.jtrangeo.2006.05.002. Journal of Transport Geography, 15(3), 151-160 (2007)

Sá, C., Florax, R. y Rietveld, P. Determinants of the regional demand for higher education in The Netherlands: a gravity model approach. doi: http://dx.doi.org/10.1080/03434002000213905. Regional Studies, 38(4), 375$392(2003)$

Sá, C., Florax, R. y Rietveld, P. Does Accessibility to Higher Education Matter? Choice Behaviour of High School Graduates in the Netherlands. doi: http://dx.doi.org/10.1080/17421770601009791. Spatial Economic Analysis, 1(2), 155-174 (2005)

Schürmann, C., Spiekermann, K. y Wegener, M. Accessibility indicators. Berichte aus dem Institut für Raumplanung, 39, IRPUD, Dortmund (1999)

Segui, J. y Petrus, J. Geografía de Redes y Sistemas de Transporte. Ed. Síntesis, Madrid, España (1991)

Talen, E. School, Community, and Spatial Equity: An Empirical Investigation of Access to Elementary Schools in West Virginia. doi: 10.1111/0004-5608.00254. Annals of the Association of American Geographers, 91(3), 465-486 (2001)

Venegas, F. y Rojas, R. Teoría y Práctica del Ordenamiento y Manejo Sustentable del Territorio: TijuanaRosarito-Tecate, Baja California, México, doi:10.1612/inf.tecnol.4077it.08, Inf. Tecnol. 20(3), 73-87 (2009)

Zhu, X. y Liu, S. Analysis of the impact of the MRT system on accesibility in Singapore using an integrated GIS tool, doi:10.1016/j.jtrangeo.2003.10.003. Journal of Transport Geography, 4(12), 89-101 (2004) 